\title{
Qualidade de vida relacionada a saúde dos idosos no município de Araguari - MG
}

DOI: $10.47224 /$ rm.v5i10.135

\author{
Mariana Fernandes Boccanera ${ }^{1}$ \\ Cássio José da Silva ${ }^{1}$ \\ Elivane Alves da Cunha ${ }^{1}$ \\ Gabriela Pereira Silva ${ }^{1}$ \\ Helder Lima Gonçalves ${ }^{1}$ \\ Isabella Pineli Chaveiro de Azevedo ${ }^{1}$ \\ Isabella Souza e Faria ${ }^{1}$ \\ Jardel Barreto Pires Amaral ${ }^{1}$ \\ João Paulo de Araújo Pelegrini ${ }^{1}$ \\ Leticia Dornelis Candido ${ }^{1}$ \\ Iara Guimarães Rodrigues ${ }^{2}$ \\ Márcio Aurélio da Silva \\ ${ }^{1}$ Discentes - Centro Universitário IMEPAC Araguari \\ ${ }^{2}$ Orientador(a) - Centro Universitário IMEPAC Araguari \\ e-mail: mariboccanera18@gmail.com
}

\section{Resumo}

Esse estudo teve por objetivo descrever a qualidade de vida relacionada a saúde da população idosa residente no município de Araguari/MG e associar com a interferência de fatores demográficos e socioeconômicos. Os dados foram obtidos através do instrumento genérico de qualidade de vida "Medical Outcomes Study 36-Item Short-Form Health Survey" (SF-36), que consiste em questionamentos a respeito do estado geral de saúde, vitalidade, capacidade funcional, dor, saúde mental, aspectos físicos, aspectos sociais e aspectos emocionais, com escalas de respostas de 0 a 100 , onde 0 corresponde a pior qualidade e 100 a melhor qualidade no domínio. O instrumento foi aplicado para a comunidade idosa (60 anos ou mais) residente na área urbana da cidade de Araguari/MG. A amostra final contemplou 365 idosos, perfazendo aproximadamente $1,6 \%$ da população. Do total analisado, evidenciou-se que $60,8 \%$ eram do sexo feminino, com renda média de 1 salário-mínimo, 52,1\% são casados, 38,1\% são aposentados, pior média geral nos escores do SF36 para o sexo feminino, pior avaliação obtida nos domínios capacidade funcional e dor, melhores resultados obtidos no domínio aspectos sociais e aspectos físicos. A média geral dos domínios do SF-36 foi 63,5 pontos, fato que comprova um regular estado ou uma regular percepção global da qualidade de vida e saúde dos idosos no município de Araguari-MG, sendo importante que haja implementação de ações de saúde abrangentes relacionadas ao envelhecimento da população, com objetivo de melhorar a qualidade de vida dos idosos.

Palavras-chave: Qualidade de vida; Saúde do idoso; Epidemiologia.

\section{Abstract}

This study aimed to describe the health-related quality of life of the elderly population living in the city of Araguari-MG and to associate it with the interference of demographic and socioeconomic factors. The data were obtained through the generic instrument of quality of life "Medical Outcomes Study 36-Item Short-Form Health Survey" (SF-36), which consists of questions about general health, vitality, functional capacity, pain, mental health, physical aspects, social aspects and emotional aspects, with scales of answers from 0 to 100, where 0 corresponds to the worst quality and 100 the best quality in the domain. The instrument was applied to the elderly community (60 years old or more) living in the urban area of the city of Araguari-MG. The final sample consisted of 365 elderly people, representing approximately $1.6 \%$ of the population. Of the total analyzed, $60.8 \%$ were female, with an average income of 1 minimum salary, 52.1\% are married, $38.1 \%$ are retired, a worse general average in SF-36 scores for female, worse evaluation obtained in the domains functional capacity and pain, better results obtained in the domain social aspects and physical aspects. The overall mean 
of the SF-36 domains was 63.5 points, a fact that confirms a regular state or a regular global perception of the quality of life and health of the elderly in the city of Araguari-MG, and it is important to implement health actions aging of the population, with the aim of improving the quality of life of the elderly.

Keywords: $\quad$ Açai pulps. Microbiological analysis. Food contamination.

\section{INTRODUÇÃO}

O aumento da expectativa de vida e o envelhecimento populacional no Brasil e no mundo têm ocorrido de forma acelerada, o que torna importante refletir sobre em que condições os idosos estão envelhecendo. Sabe-se que o envelhecimento é um processo complexo, intersetorial, que abarca discussões da área da saúde, educação, assistência social, previdência e habitação, incluindo-se os aspectos sociais e econômicos que afetam a qualidade de vida da população que envelhece (OLIVEIRA, 2014).

O envelhecimento pode ser definido como um conjunto das modificações que decorrem do avançar da idade para além da fase da maturidade. É, de certa maneira, um processo inverso do desenvolvimento: neste ocorre o crescimento do ser vivo, com aparição progressiva de características de base genética próprias de cada indivíduo. Após um ponto em que o desenvolvimento atinge o seu máximo, começa a observar-se a diminuição progressiva das aptidões e capacidades, tanto físicas como mentais: é a involução, em contraponto com a evolução que a precedeu (BARRETO, 2017).

O Brasil vem tentando se preparar para responder as crescentes demandas de sua população que envelhece, por meio de ações e políticas implementadas com a finalidade de garantir os direitos a saúde dos idosos e um envelhecimento com Qualidade de Vida (QV), (SCHMIDT, 2011).

Apesar do processo de envelhecimento não estar, necessariamente, relacionado a doenças e incapacidades, percebe-se que as doenças crônicodegenerativas são frequentemente encontradas nos idosos (CHAIMOWICZ, 1997). Assim, a tendência atual é termos um número crescente de indivíduos idosos que, apesar de viverem mais, apresentam maiores condições crônicas, e percebe-se que o aumento desse número de doenças crônicas está diretamente relacionado com uma maior incapacidade funcional (ALVES, 2007).
Entre as causas dos problemas de saúde mais frequentes nos idosos, citam-se sobretudo a interação de fatores genéticos e hábitos não saudáveis, como uma dieta não balanceada, tabagismo, etilismo e sedentarismo (TONELLI, 2014).

Evidencia-se, na atualidade, um predomínio de acometimento por doenças crônicas não degenerativas, que se constituem em importante causa de morte e representam um problema de saúde de alta magnitude (SCHMIDT, 2011). Além disso, as doenças crônicas não transmissíveis são as principais causas de morte no mundo, além de prejudicar a qualidade de vida (QV) e desencadear limitações para as atividades de vida diária (AVD) dos idosos (TONELLI, 2014).

A qualidade de vida relacionada a saúde é particularmente importante na terceira idade, e, sobretudo, em idosos em situação vulnerável. 0 estado de saúde tem influência significativa na sua QV percebida, bem como sobre o seu funcionamento cognitivo e social. Os modelos de QV vão desde a satisfação com a vida, ao bem-estar social, a modelos baseados em conceitos de independência, controle, competências sociais e cognitivas. Nos dias de hoje, este constructo encontra-se, também, relacionado com dimensões menos tangíveis, como é o caso do sentido de segurança, da dignidade pessoal, das oportunidades de atingir objetivos pessoais, da satisfação com a vida, da alegria e do sentido positivo de si (SOUSA, et al., 2003).

Dessa forma, este trabalho tem como objetivo principal dimensionar o perfil da Qualidade de Vida relacionada a Saúde (QVRS) da população idosa do município de Araguari. Assim, foram relacionados fatores demográficos e socioeconômicos desse município, por meio do instrumento genérico de qualidade de vida "Medical Outcomes Study 36-Item Short-Form Health Survey" (SF-36). 
Trata-se de um estudo quantitativo, descritivo de corte transversal. A amostra obtida foi composta por 365 indivíduos de um total de 57.817 propostos, perfazendo aproximadamente $0,662 \%$ da população. Dessa forma, o instrumento genérico de qualidade de vida "Medical Outcomes Study 36-Item Short-Form Health Survey" (SF-36) versão brasileira (CICONELLI et al., 1997) e questionário sociodemográfico foram aplicados à população idosa acima de 60 anos residentes na área urbana do munícipio de AraguariMG.

O instrumento SF-36 consiste em uma escala utilizada para avaliação da qualidade de vida relacionada a saúde através de domínios como estado geral de saúde, vitalidade, capacidade funcional, dor, saúde mental, aspectos físicos, aspectos sociais e aspectos emocionais. Para análise dos domínios, verifica-se que a pontuação de cada quesito varia de 0 a 100, onde 0 corresponde a pior qualidade e 100 a melhor qualidade no domínio. Já o questionário socioeconômico foi obtido através das variáveis mais pesquisadas em artigos publicados referentes à temática e que seriam de interesse para o estudo, sendo abordado sexo, idade, sexo, estado civil, renda e ocupação.

Foram incluídos na pesquisa aqueles idosos que possuíam aptidão cognitiva para responder ao questionário, excluindo-se aqueles com confusão, desorientação ou déficit mental.

Os dados foram analisados descritivamente, com a determinação de valores absolutos e relativos, expressos em média e desvio padrão, organizado em tabelas, com o auxílio do software Excel $^{\circledR}$ e BioStat 5.3, utilizando-se dos testes Mann-Whitney, ANOVA de Kruskal-Wallis/ Post-hoc Teste Dunn.

O presente estudo foi submetido e aprovado pelo Comitê de Ética em pesquisa sob o protocolo de número 126151/2017.

\section{RESULTADOS}

A população foi composta por 365 idosos residentes em área urbana do Município de Araguari/MG, sendo a maioria $(49,1 \%)$ da faixa etária de 60 a 69 anos e do sexo feminino $(60,8 \%)$. Em relação ao estado civil mais da metade $(52,1 \%)$ são casados e com renda de até um salário-mínimo ( $\mathrm{R} \$ 954)$. Encontrou-se maior prevalência de idosos que não trabalham ou estavam desempregados $(38,1 \%)$, seguido daqueles que não souberam referir sua ocupação $(35,3 \%)$.

Referente aos resultados dos domínios avaliados pelo questionário SF-36, apresentados na tabela 1, verifica-se de modo geral uma tendência de diferença estatisticamente significativa principalmente para o sexo feminino, mostrando resultados de escores menores em comparação com o sexo masculino.

Tabela 1 - Média dos escores do SF-36 estratificado por sexo. Araguari - MG. 2018.

\begin{tabular}{|c|c|c|c|c|}
\hline \multirow{3}{*}{ Domínios SF-36 } & \multicolumn{3}{|c|}{ Média (IC 95\%) } & \multirow{3}{*}{ Valor $p$} \\
\hline & Total & Masculino & Feminino & \\
\hline & $n=365$ & $n=143$ & $n=222$ & \\
\hline Aspectos físicos & $72(69-74)$ & $76(72-80)$ & $69(65-73)$ & $0,0123^{*}$ \\
\hline Capacidade Funcional & $55(50-59)$ & $60(53-67)$ & $51(45-57)$ & 0,0768 \\
\hline Dor & $61(58-64)$ & $67(62-72)$ & $57(53-62)$ & $0,0065^{*}$ \\
\hline Estado geral de saúde & $62(60-64)$ & $66(63-69)$ & $59(56-62)$ & $0,0034^{*}$ \\
\hline Vitalidade & $64(62-67)$ & $67(64-71)$ & $62(59-65)$ & $0,0373^{*}$ \\
\hline Aspectos sociais & $75(72-77)$ & $79(75-83)$ & $72(68-76)$ & $0,0448^{*}$ \\
\hline Aspectos emocionais & $62(58-67)$ & $70(63-77)$ & $58(52-64)$ & $0,0167^{*}$ \\
\hline Saúde mental & $67(65-69)$ & $71(68-74)$ & $64(62-67)$ & $0,0007^{*}$ \\
\hline
\end{tabular}

Teste Mann-Whitney; *diferença estatisticamente significativa; $p<0,05$

Fonte: os autores

Observa-se ainda que a melhor média foi obtida no domínio aspectos sociais para ambos os sexos e que a pior avaliação foi obtida no domínio capacidade funcional, indo de encontro ao observado no escore dor e contrapondo-se aos bons escores médios observados no domínio aspectos físicos (72). Importante ressaltar que o componente capacidade funcional não apresentou significância estatística quando analisado a média de escores gerais. 
A média dos escores por domínio e faixa etária apresentados na tabela 2 , evidencia que idosos com idade maior a 80 anos obtiveram pior resposta no quesito capacidade funcional, estando em concordância com os valores observados entre idosos na faixa etária de 60 a 74 anos, em contrapartida o melhor escore obtido foi entre a faixa etária de 75 a 79 anos. Em relação ao domínio atividades sociais, observou-se melhor pontuação (79) nos idosos com idade superior a 80 anos e menor pontuação entre os indivíduos com 70 a 74 anos e resultado semelhante também se observa no domínio saúde mental. Dentre todos os domínios, o que apresentou melhor pontuação foi a atividade física na faixa etária entre 60 a 69 anos. Inversamente, o domínio em que predomina a pior pontuação foi a limitação da função física, principalmente entre idosos maiores de 80 anos, indo de encontro aos valores apresentados no escore dor.

Tabela 2 - Média dos escores do SF-36 estratificado por faixa etária. Araguari/MG. 2018.

\begin{tabular}{|c|c|c|c|c|c|c|}
\hline \multirow{2}{*}{$\begin{array}{l}\text { Domínios SF-36 } \\
\text { (IC 95\%) }\end{array}$} & \multicolumn{5}{|c|}{ Faixa Etária } & \multirow{2}{*}{ Valor $p$} \\
\hline & 60 a 64 & 65 a 69 & 70 a 74 & 75 a 79 & 80 ou mais & \\
\hline Aspectos físicos & $76,2(70,3-82,1)$ & $76,5(76,5-81,5)$ & $70,6(64,3-6,8)$ & $69,9(63,1-76,7)$ & $55,4(47,1-63,7)$ & $<0,0001^{*}$ \\
\hline $\begin{array}{l}\text { Capacidade } \\
\text { Funcional }\end{array}$ & $51,1(41,6-60,7)$ & $56,0(47,5-64,5)$ & $55,2(46,1-64,4)$ & $67,6(56,3-78,9)$ & $40,5(27,7-53,3)$ & $0,0490 *$ \\
\hline Dor & $62,3(55,2-69,3)$ & $62,7(56,4-69,0)$ & $61,5(54,5-68,5)$ & $62,7(54,8-70,5)$ & $51,2(41,8-60,6)$ & 0,2797 \\
\hline $\begin{array}{l}\text { Estado geral de } \\
\text { saúde }\end{array}$ & $62,6(58,6-66,6)$ & $63,0(59,0-67,0)$ & $62,8(58,1-67,5)$ & $62,5(56,7-68,3)$ & $57,0(50,5-63,6)$ & 0,5858 \\
\hline Vitalidade & $62,2(56,9-67,6)$ & $66,2(61,9-70,5)$ & $67,0(61,9-72,0)$ & $65,4(59,1-71,6)$ & $56,7(49,6-63,7)$ & 0,1806 \\
\hline Aspectos sociais & $74,8(69,1-80,4)$ & $74,8(69,2-80,5)$ & $72,3(66,3-78,4)$ & $76,3(69,6-82,9)$ & $79,0(73,2-84,7)$ & 0,9729 \\
\hline $\begin{array}{l}\text { Aspectos } \\
\text { emocionais }\end{array}$ & $63,2(53,9-72,4)$ & $61,6(53,9-72,4)$ & $65,8(56,6-75,0)$ & $62,2(50,8-73,7)$ & $55,5(41,1-69,9)$ & 0,8008 \\
\hline Saúde mental & $63,2(59,0-67,4)$ & $66,1(62,5-69,7)$ & $66,7(62,7-7,08)$ & $72,0(67,6-76,3)$ & $68,9(64,1-73,7)$ & 0,1168 \\
\hline
\end{tabular}

Tabela 3 - Média dos escores do SF-36 estratificado por faixa etária no sexo feminino. Araguari/MG.2018

\begin{tabular}{|c|c|c|c|c|c|c|}
\hline \multirow{2}{*}{$\begin{array}{l}\text { Domínios SF-36 } \\
\text { (IC 95\%) }\end{array}$} & \multicolumn{5}{|c|}{ Faixa Etária - Sexo Feminino } & \multirow{2}{*}{ Valor $p$} \\
\hline & 60 a 64 & 65 a 69 & 70 a 74 & 75 a 79 & 80 ou mais & \\
\hline Aspectos físicos & $72,6(65,2-80,0)$ & $74,3(67,2-81,3)$ & $65,1(56,4-73,7)$ & $64,4(55,9-72,9)$ & $57,4(46,2-68,6)$ & $0,0168^{*}$ \\
\hline $\begin{array}{l}\text { Capacidade } \\
\text { Funcional }\end{array}$ & $51,3(39,5-63,0)$ & $52,4(41,7-63,1)$ & $48,4(36,2-60,6)$ & $62,9(47,2-78,5)$ & $30,3(12,1-48,5)$ & 0,1777 \\
\hline Dor & $58,9(50,0-67,7)$ & $61,3(53,6-69,1)$ & $51,0(41,6-60,3)$ & $61,2(50,9-71,4)$ & $49,1(33,9-64,3)$ & 0,3069 \\
\hline $\begin{array}{l}\text { Estado geral de } \\
\text { saúde }\end{array}$ & $61,2(54,2-67,9)$ & $60,5(55,4-65,6)$ & $57,1(50,2-64,1)$ & $61,1(52,7-69,5)$ & $52,0(41,6-62,4)$ & 0,5165 \\
\hline Vitalidade & $61,1(54,2-67,9)$ & $65,3(59,9-70,7)$ & $63,1(56,3-69,8)$ & $60,1(51,5-68,7)$ & $56,1(45,9-66,2)$ & 0,6159 \\
\hline Aspectos sociais & $72,3(64,8-79,7)$ & $74,6(67,3-82,0)$ & $66,8(58,2-75,4)$ & $71,3(62,3-80,3)$ & $80,7(73,6-87,8)$ & 0,7320 \\
\hline $\begin{array}{l}\text { Aspectos } \\
\text { emocionais }\end{array}$ & $63,8(52,6-75,0)$ & $56,1(45,3-67,0)$ & $56,6(43,9-69,4)$ & $56,1(41,2-70,9)$ & $49,1(27,6-70,5)$ & 0,7461 \\
\hline Saúde mental & $61,4(56,2-66,6)$ & $64,8(60,1-69,5)$ & $61,9(56,2-67,6)$ & $67,8(62,1-73,4)$ & $69,7(62,5-76,8)$ & 0,3762 \\
\hline
\end{tabular}

ANOVA de Kruskal-Wallis/Post-hoc Teste Dunn;

* diferença estatisticamente significativa;

$\mathrm{p}<0,05$

Fonte: os autores 
Em relação aos escores médios do SF-36 estratificados por faixa etária no sexo feminino, observado na tabela 3, a menor média entre os escores foi observado no domínio capacidade funcional, principalmente na faixa etária de maiores de 80 anos, indo de encontro aos valores observados no quesito dor. A maior média dos escores foi obtida no domínio aspectos sociais, que demonstrou resultado inverso ao observado no domínio capacidade funcional, com melhor resultados na população maior de 80 anos. 0 único domínio que não houve diferença estatisticamente significativa foi a capacidade funcional dos idosos do sexo feminino.
Na tabela 4, encontra-se a pontuação média do SF36 no sexo masculino, estratificado por faixa etária. Pode-se observar que os idosos de Araguari apresentam piores resultados no quesito capacidade funcional, com escores semelhantes entre a faixa etária 60 a 64 anos e 80 anos e mais, indo de encontro às menores pontuações para o escore aspectos físicos. Ademais, observa-se bons resultados na faixa etária geral para os domínios aspectos sociais e aspectos emocionais, que vai de encontro aos bons escores observados na saúde mental. Referente ao domínio estado geral de saúde, observa-se bons valores médios semelhantes na faixa etária geral. Os piores resultados globais são observados na faixa etária maior de 80 anos.

Tabela 4 - Média dos escores SF-36 estratificado por faixa etária no sexo masculino. Araguari/MG.2018

\begin{tabular}{|c|c|c|c|c|c|c|}
\hline \multirow{2}{*}{$\begin{array}{l}\text { Domínios SF-36 } \\
\text { (IC 95\%) }\end{array}$} & \multicolumn{5}{|c|}{ Faixa Etária - Sexo Masculino } & \multirow{2}{*}{ Valor $p$} \\
\hline & 60 a 64 & 65 a 69 & 70 a 74 & 75 a 79 & 80 ou mais & \\
\hline Aspectos físicos & $83,9(74,8-93,1)$ & $80,4(74,3-86,5)$ & $77,2(68,4-85,9)$ & $80,0(69,9-90,1)$ & $53,7(41,5-65,9)$ & 0,0004 \\
\hline $\begin{array}{l}\text { Capacidade } \\
\text { Funcional }\end{array}$ & $50,9(34,3-67,5)$ & $62,5(48,4-76,6)$ & $63,5(49,9-77,0)$ & $76,3(62,1-90,6)$ & $48,9(31,4-66,5)$ & 0,2571 \\
\hline Dor & $69,6(58,4-80,8)$ & $65,1(54,2-76,0)$ & $74,2(65,0-83,4)$ & $65,4(53,4-77,4)$ & $53,0(41,0-64,9)$ & 0,0712 \\
\hline $\begin{array}{l}\text { Estado geral de } \\
\text { saúde }\end{array}$ & $65,7(58,9-72,5)$ & $67,6(61,4-73,7)$ & $69,5(64,0-75,1)$ & $65,0(59,0-71,0)$ & $61,2(53,0-69,4)$ & 0,5349 \\
\hline Vitalidade & $64,6(56,3-73,0)$ & $67,8(60,4-75,2)$ & $71,7(64,3-79,1)$ & $75,0(68,7-81,3)$ & $57,2(47,3-67,1)$ & 0,0976 \\
\hline Aspectos sociais & $80,1(72,8-87,4)$ & $75,2(66,4-84,0)$ & $79,0(7,09-87,2)$ & $85,5(77,5-93,5)$ & $77,5(68,8-86,2)$ & 0,6453 \\
\hline $\begin{array}{l}\text { Aspectos } \\
\text { emocionais }\end{array}$ & $61,8(45,1-78,5)$ & $71,5(57,1-85,9)$ & $76,9(64,4-89,4)$ & $73,5(56,5-90,5)$ & $60,8(41,3-80,4)$ & 0,5657 \\
\hline Saúde mental & $67,1(60,3-74,0)$ & $68,4(62,7-74,0)$ & $72,6(67,3-77,9)$ & $79,7(74,3-85,1)$ & $68,2(61,6-74,8)$ & 0,0554 \\
\hline
\end{tabular}

ANOVA de Kruskal-Wallis/Post-hoc Teste Dunn;

*diferença estatisticamente significativa;

$\mathrm{p}<0,05$

Fonte: os autores

\section{DISCUSSÃO}

Após análise dos resultados, a pesquisa aponta que a maioria dos participantes $(60,82 \%)$ são do sexo feminino, que vai de encontro ao verificado por Camelo et al. (2016), Faria et al. (2011) e Cordeiro et al. (2014). Esse dado confirma uma certa desigualdade de gênero na expectativa de vida, onde há uma maior prevalência na proporção de mulheres em relação aos homens. Santos (2014) afirma que no Brasil as mulheres vivem em média oito anos a mais que os homens, e expõe alguns fatores que podem ser utilizados para este achado, destacando-se: proteção de fatores biológicos, as diferentes exposições aos fatores ambientais, as diferenças no uso e abuso de drogas lícitas e a melhor busca aos serviços de saúde.

Percebe-se ainda que grande parte dos entrevistados são idosos casados $(52,05 \%)$ e pertencentes à faixa etária de 65-69 anos (26,03\%). Tais resultados também vão ao encontro do estudo de Faria (2011) que, além de ter encontrado uma prevalência de idade dentro dessa mesma faixa etária, descreveu que $70 \%$ de da população em estudo eram casados. Infere-se, dessa forma, que com o avanço da idade, há um predomínio da morbimortalidade e quanto mais jovens menos complicações de saúde apresentam. Dessa forma, presente estudo 
assemelha-se com o estudo de Campos (2016) inferindo que o casamento pode ser um fator relevante para o envelhecimento saudável, portanto a relação conjugal é uma forma de prevenir a dependência funcional.

Quando analisada a ocupação e renda houve um predomínio de $38,8 \%$ de idosos que não trabalham ou estão desempregados com renda média inferior a quatro salários-mínimos, o que pressupõe que maioria dos idosos são aposentados pela faixa etária pertencentes. Tais resultados se correlacionam com o artigo de Dias (2017) no qual $88,6 \%$ dos idosos são aposentados e $11,4 \%$ são pensionistas. Da mesma forma, no estudo de Camelo (2016), 82\% dos idosos entrevistados informaram ter renda familiar inferior a quatro salários mínimos. $O$ resultado de ambos os estudos evidenciou a baixa condição monetária dos entrevistados, dado que pode influenciar negativamente seu estado de saúde, pois idosos que possuem maior renda tem acesso mais fácil a serviços de saúde, medicamentos, atividades de laser, dentre outros. Dessa forma, Boing (2013) identificou em seu estudo que a migração dos usuários do SUS para o setor privado ou de copagamento para garantir o acesso aos medicamentos implica maior comprometimento de renda nos gastos em saúde, extremamente elevados, que penalizam, sobretudo, os mais pobres.

Ao analisar os domínios do questionário SF-36, verifica-se que o sexo feminino apresenta os piores resultados, em especial nos quesitos emocionais, saúde mental e aspectos sociais, uma vez que a percepção do envelhecimento para as mulheres idosas jovens, num primeiro momento, promove um certo desgaste emocional. Pereira (2006), evidencia essa percepção em seus resultados quando relata que a mulher idosa se apresenta mais vulnerável quando comparada aos homens em relação aos aspectos físicos e mentais, o que de certa forma justifica os menores scores nos domínios que envolvem a perspectiva psicológica.

Os comprometimentos da função motora e da aptidão física associados ao processo de envelhecimento contribuem negativamente a QVRS, evidentes na menor média de pontos no domínio capacidade funcional, além da piora dos escores dos aspectos físicos com a progressão das décadas. De acordo com Joia (2007) é a dor física que instiga, consome e enfraquece, expondo suas fragilidades e ameaçando sua segurança, autonomia e independência, impedindo muitas vezes sua capacidade de realizar as atividades da vida diária, fatores que interferem consideravelmente sua qualidade de vida, sendo mais acentuado no sexo feminino.

O domínio atividades Sociais apresentou o maior escore médio entre os domínios. Essa superioridade pode estar relacionada às ações de políticas públicas disponíveis na rede de assistência social ao idoso no município de Araguari-MG, que dispõe de um Centro de Convivência do Idoso. $O$ espaço integra atividades culturais e recreativas que proporcionam um envelhecimento saudável, principalmente em idosos maiores de 80 anos, com práticas de exercícios, oficinas e cursos que visam o fortalecimento de laços interpessoais, prevenindo isolamento social e valorização da autoestima.

Em relação ao domínio vitalidade, assim como saúde geral, verifica-se que quanto maior o vigor demonstrado pelos idosos para realizar atividades diárias, melhor será o seu estado geral de saúde, o que resulta em uma melhor qualidade de vida. Dessa forma, os escores médios apresentados no domínio vitalidade mostra-se inferior à média do estudo feito por Pereira (2012), inclusive no domínio saúde geral, que foi o melhor resultado entre os domínios no seu estudo. Os dados descritos no estudo de Pereira (2012) contrapõem-se aos apresentados neste estudo, uma vez que vitalidade e saúde geral apresentaram escores divergentes, sendo saúde geral o maior score e vitalidade o menor score do estudo. Em contrapartida, neste estudo, saúde geral e vitalidade apresentam semelhança de resultados, o que mostra que são domínios interligados e diretamente relacionados.

A saúde mental avalia itens como ansiedade, depressão, alterações do comportamento, descontrole emocional e bem-estar psicológico. 0 presente estudo revelou uma média de pontuação superior ao estudo feito por Oliveira (2009), o que implica positivamente na QV dos idosos, sendo que a presença de incapacidades e instalação de quadros de dependência promovem um declínio funcional, com redução e perda das habilidades para a realização das atividades da vida diária.

\section{CONCLUSÕES}

A média geral de todos os domínios avaliados com o SF-36 foi 63,5 pontos, fato que comprova um regular estado ou uma regular percepção global da qualidade de vida e saúde dos idosos no município de AraguariMG. Levando-se em consideração a multidimensionalidade representada nos diversos domínios do instrumento SF-36 e a alta prevalência 
de idosos nessa cidade percebe-se a necessidade da implementação de ações e investimento em áreas sociais visando à melhoria da saúde do idoso.

O aumento da expectativa de vida da população brasileira combinado a quedas acentuadas nas taxas de fertilidade resulta em uma mudança no padrão da pirâmide demográfica do nosso país. Dessa forma, é importante que haja a implementação de ações de saúde pública abrangentes relacionadas ao envelhecimento da população com o desafio de ampliar e melhorar cada vez mais a capacidade funcional, física e mental dos idosos no município.

\section{REFERÊNCIAS}

ALVES, L.C. et al. A influência das doenças crônicas na capacidade funcional dos idosos do Município de São Paulo. Caderno de saúde pública, v.23, n.8, 2007.

ANDRADE, A.; MARTINS, R. Funcionalidade familiar e qualidade de vida dos idosos. Millenium-Journal of Education, Technologies, and Health, n. 40, p. 185199, 2016.

ARAÚJO, Denise Sardinha Mendes Soares de; ARAÚJO, Claudio Gil Soares de. Aptidão física, saúde e qualidade de vida relacionada à saúde em adultos. Revista brasileira de medicina do esporte, v. 6, n. 5, p. 194-203, 2000.

BARRETO, J. Envelhecimento e qualidade de vida: o desafio atual. Sociologia: Revista da Faculdade de Letras da Universidade do Porto, v. 15, 2017.

BOING, Alexandra Crispim, et al. Acesso a medicamentos no setor público: análise de usuários do Sistema Único de Saúde no Brasil. Caderno de Saúde Pública, v. 29, p. 691-701, 2013

CAMELO, Lidyane do Valle; GIATTI, Luana; BARRETO, Sandhi Maria. Qualidade de vida relacionada à saúde em idosos residentes em região de alta vulnerabilidade para saúde de Belo Horizonte, Minas Gerais. Revista Brasileira de Epidemiologia, v. 19, p. 280-293, 2016.

CAMPOS, Ana Cristina Viana et al. Perfil do envelhecimento saudável de idosos brasileiros octogenários. Revista Latino-Americana de Enfermagem, v. 24, p. 1-11, 2016.

CARDOSO, F. P.; GONÇALVES, A. K. Associação entre domínio de capacidade funcional (SF-36), medo de cair e histórico de quedas em idosos ativos. Ciência em Movimento, v. 18, n. 36, p. 1-8, 2016.

CELICH, Kátia Lilian Sedrez; GALON, Cátia. Dor crônica em idosos e sua influência nas atividades da vida diária e convivência social. Revista Brasileira de Geriatria e Gerontologia, v. 12, n. 3, p. 345-359, 2009.

CHAIMOWICZ, F. A saude dos idosos brasileiros as vesperas do seculo XXI: problemas, projeçoes e alternativas. Rev. Saúde Pública, v.31, n. 2, p. 184200, 1997.

CICONELLI, Rozana Mesquita. Tradução para o português e validação do questionário genérico de avaliação de qualidade de vida Medical Outcomes Study 36-Item Short-Form Health Survey (SF-36). 1997.

CORDEIRO, Juliana et al. Efeitos da atividade física na memória declarativa, capacidade funcional e qualidade de vida em idosos. Revista Brasileira de Geriatria e Gerontologia, v. 17, n. 3, p. 541-552, 2014.

DE OLIVEIRA, Luciane Criado; PIVOTO, Ercília Aparecida; VIANNA, Patrícia Canteruccio Pontes. Análise dos resultados de qualidade de vida em idosos praticantes de dança sênior através do SF36. Acta Fisiátrica, v. 16, n. 3, p. 101-104, 2009.

DIAS, Ernandes Gonçalves et al. ESTILO DE VIDA DE IDOSOS USUÁRIOS DE UMA UNIDADE BÁSICA DE SAÚDE. Arquivos de Ciências da Saúde da UNIPAR, v. 21, n. 2, 2017.

FARIA, C. D. C. M. et al. Comparação dos instrumentos de qualidade de vida Perfil de Saúde de Nottingham e Short Form-36 em idosos da comunidade. Rev. bras. fisiote. São Carlos, v. 15, n. 5, p. 399-405, 2011.

FECHINE, A. O processo de envelhecimento: as principais alterações que acontecem com o idoso com o passar dos anos. Revista científica internacional, v. 1, n. 7, 2012.

FRAIMAN, A. P. Coisas da Idade. São Paulo: Alexa Cultural, 2004.

GOTTLIEB, M.G.V, SCHWANKE, C.H.A, GOMES I, CRUZ, I.B.M. Envelhecimento e longevidade no Rio Grande do Sul: um perfil histórico, étnico e de morbi-mortalidade dos idosos. Revista Brasileira de Geriatria e Gerontologia, v. 14, n.2, p. 365-380. 2011 
HE, W.; GOODKIND, D.; KOWAL, P. An aging world: 2015. Washington, DC: United States Census Bureau, 2016. 165 p. (International population reports). Disponível em:

https://www.census.gov/content/dam/Census/librar $\mathrm{y} /$ publications/2016/demo/p95-16-1.pdf. Acesso em: 17 ago. 2017.

INDICADORES IBGE. Características da população e dos domicílios: População residente, por cor ou raça, segundo o sexo e os grupos de idade - Brasil - 2010. Rio de Janeiro: IBGE, 2010. Disponível em: <ftp://ftp.ibge.gov.br/Censos/Censo_Demografico_2 010/Resultados_do_Universo/xls/Brasil/tab1_3_1.zi p>. Acesso em: 26.ago. 2017.

JOIA, Luciane Cristina; RUIZ, Tania; DONALISIO, Maria Rita. Condições associadas ao grau de satisfação com a vida entre a população de idosos. Revista de Saúde Pública, v. 41, p. 131-138, 2007.

LEITE F.C; CONCEIÇÃO, A; COELHO, M.R. Políticas públicas para a saúde do idoso: revisão sistemática. Revista Brasileira de Enfermagem, v. 63, n. 2, 2010.

LEITE, MarinêsTambara et al. Qualidade de vida e nível cognitivo de pessoas idosas participantes de grupos de convivência. Rev. Bras. Gerontol.v.15, n.3, p. 481-492, 2012. Disponível em:

>http://www.scielo.br/scielo.php?pid=S1809982320 $12000300009 \&$ script=sci abstract\&tlng=pt. Acesso em: 07 nov. 2018.

MARTINS, R; MESTRE, M. Esperança e qualidade de vida em idosos. Millenium-Journal of Education, Technologies, and Health, n. 47, p. 153-162, 2016.

OLIVEIRA, A.D; RAMOS, A.O; PANHOCA, l; ALVES, V.L.S. A intersetorialidade nas políticas públicas para o envelhecimento no Brasil. Rev Kairós Gerontol. Disponível em:

<http://revistas.pucsp.br/index.php/kairos/article/vi ew/21278/15556>. Acesso em: 28 ago. 2017.

PEREIRA, Eduardo da Silva; BORGES, Ítalo Soares. Análise da percepção geral de saúde e da qualidade de vida de idosos praticantes de hidroginástica a partir do instrumento SF-36. RBPFEX-Revista Brasileira de Prescrição e Fisiologia do Exercício, v. 5, n. 27, 2012.

PEREIRA, Renata Junqueira et al. Contribuição dos domínios físico, social, psicológico e ambiental para a qualidade de vida global de idosos. Rev Psiquiatr Rio Gd Sul, v. 28, n. 1, p. 27-38, 2006.

PIMENTA, J.R.; Navarro, F. A qualidade de vida e o bem-estar dos idosos: uma análise comparativa entre sedentários e praticantes de exercício físico através do protocolo SF-36. Revista Brasileira de Prescrição e Fisiologia do Exercício. Vol. 3. Num. 15. 2009. p. 295-301.

SANTOS, Gerson Souza; CUNHA, Isabel Cristina Kowal OIm. Avaliação da qualidade de vida de mulheres idosas na comunidade. Revista de Enfermagem do Centro-Oeste Mineiro, 2015.

SCHMIDT, M.I; DUNCAN, B.B; AZEVEDO, G.S; MENEZES, A.N; MONTEIRO, C.A; BARRETO, S.M et al. Chronic non communicable diseases in Brazil: burden and current challenges. Disponível em: $<$ http://

www.thelancet.com/journals/lancet/article/PIIS0140 -6736(11)60135-9/abstract>. Acesso em: 26 ago. 2017.

SEIDL, Eliane Maria Fleury; ZANNON, Célia Maria Lana da Costa. Qualidade de vida e saúde: aspectos conceituais e metodológicos. Cadernos de saúde pública, v. 20, p. 580-588, 2004.

SILVA, Patrick Leonardo Nogueira et al. Avaliação da qualidade de vida de idosos praticantes de atividade física de uma unidade básica de saúde de minas gerais. Rev. Da Universidade Vale do Rio Verde. V.14, n.2, p 24-35, 2016. Disponível em>http://periodicos.unincor.br/index.php/revistau nincor/article/view/2584. Acesso em: 07 nov. 2018.

SOUSA, L.; GALANTE, H; FIGUEIREDO, D. (2003). Qualidade de Vida e Bem-Estar dos idosos: um estudo exploratório na população portuguesa. Revista de Saúde Pública; 37(3):364-71.

TONELLI, M; RIELLA, M. Chronic kidney disease and the aging population. J Bras Nefrol. Disponível em: <https://www.ncbi.nlm.nih.gov/pmc/articles/P MC3968612/>. Acesso em: 07 nov. 2018.

World population prospects: the 2015 revision. New York: United Nations, Department of Economic and Social Affair, Population Division, 2015. Disponível em:

https://esa.un.org/unpd/wpp/Download/Standard/P opulation//. Acesso em 26 ago. 2017. 